\title{
Exploring ways to improve health system performance in developing countries
}

\author{
Health systems in low- and middle-income countries: An economic and policy \\ perspective \\ Richard D Smith \& Kara Hanson (Eds) \\ Oxford University Press, New York, 2012, 288 pp., \$59.95, ISBN 978199566761
}

This lively collection of articles investigating the challenges faced by health systems in developing countries is a welcome addition to the current health literature. Writing from a non-technical perspective, the authors strike a balance between deterring the general reader with excessive technical jargon and being too elementary to interest specialists. The book will thus appeal to a broad readership of health care professionals, researchers, advisors, students, and those outside the health profession involved in trade policy, economics, public policy and development.

The 12 chapters, written by local and international opinion leaders, are organised into four sections. It is structured to emphasise a holistic view of health systems that goes beyond health care delivery to include geographical, sectoral and temporal influences on health and health care.

The first section explores the health system. It explains the concept of 'health system' for the reader, helpfully defining the terms used in this field; looks at what such a system is, why it is necessary, how it works as a complex adaptive system, and what the consequences are of adopting a systems approach to health care; and examines the political and economic aspects of health care and 'systems thinking' in the context of institutions.

The second section, on financing, purchasing, provision and performance in the health sector in the broader context of the health system, covers a wide range of topics: measuring and evaluating performance (a complex task, since by definition a system cannot be quantified); the rationale for such measurement and the quality of some performance assessment frameworks; revenue collection and pooling arrangements in financing, and a way forward for developing countries; information and incentives, both very relevant for health systems since they influence the behaviour of participating agents; human resources, which essentially determine the system's efficiency; push and pull factors and the role of politic factors; and the role of pharmaceuticals in health sector expenditure, including issues of $\mathrm{R} \& \mathrm{D}$, manufacturing, regulation, financing, distribution and access, and, since pharmaceuticals are the single most important healthrelated product traded, the matter of trade and TRIPS (trade related intellectual property rights).

The third section explores health and systems in the wider context. It covers health systems and international trade, and how the systems are affected by globalisation and trade liberalisation, particularly World Trade Organisation agreements and regional and bilateral trade agreements; health systems and external financing, highlighting the poorer countries' reliance on external funding sources; the patterns of aid flows to these countries, including concerns about the effectiveness of such aid and how policies should respond to it; the health system and the wider social determinants of health and the various models used to explain these determinants; influences on the 
distribution of health outcomes; non-health sector interventions aimed at improving health and well-being, and the implications for the health sector within the wider health system; and finally the myriad of global factors that can affect the health system from both the demand and the supply side.

The final section deals with the future of health systems. It argues that global health diplomacy is the 'missing pillar' to strengthen health systems in low- and middleincome countries and that the rapid pace of global change makes it necessary to engage with non-health bodies, especially at a global policy level. The concept of 'global health diplomacy' is explored, and advice is offered on the negotiation skills that will be needed to equip role players in the health field to maximise opportunities and minimise risks.

The following were for me some of the main plus points of this book.

The introductory section on the health system lays an admirably solid foundation to help the reader benefit from the rest of the book. The first chapter should appeal to all readers, irrespective of discipline or field of work, since a systems perspective can be used to analyse life issues of all kinds, not only health-related. Understanding and accepting this fundamental systems approach to addressing health issues is extremely relevant to South Africa today, where we are on the brink of a major health reform, the implementation of National Health Insurance (NHI).

I liked the emphasis on the need for a paradigm shift in the way we view health issues, especially the succinct observation that 'learning (inherent to systems) is more about problem-coping than problem solving' (p. 38). These authors argue that most approaches to health care problems are reductionist since they implicitly 'problemsolve' rather than 'problem-cope'. The approaches they recommend for dealing with problems are experimentation, diversity, reflection - and embracing not only success but also failure (pp. 39-42).

The discussion of measure versus meaning interested me (pp. 64-9). We are reminded of Einstein's dictum that 'not everything that can be counted counts, and not everything that counts can be counted'. This is true in the context of all systems, especially a health care system. The authors advocate that health systems role players should move beyond the exclusive focus on indicators and towards understanding the issues that lie beneath. They point out that measuring performance does not necessarily improve performance, and they cite cases where excessive focus on indicators may lead to neglect of other areas of work (p. 69).

This ties in well with the important matter of incentives, which is elaborated on in the second section. The authors note that the impact of incentives on the delivery of health services is extremely relative in the context of health systems. They explore the positive and the unintended effects of incentives (p. 112) and the way that changing rules and institutions can result in either progressive or regressive health outcomes. They emphasise that changes made to incentives in an environment fraught with information problems are done mostly on a small scale and not reviewed over the long term, making it hard to observe the adaptive behaviour of participating agents within a system. They recommend that incentive interventions are best undertaken from a systems approach. 
On the minus side, there is a gap to be filled - no chapter focuses on the role of the patient per se in the health system, although this role is implied in the discussion of certain frameworks. I also highlighted the following issues that the editors and the authors might want to clarify in a subsequent edition of this book.

The separation of the health sector from the health system is done mostly for reasons of practicality. However, we should remember that the health sector is a subsystem of the health system and therefore must possess the inherent characteristics of a system. In some chapters I felt the systems approach did not flow through strongly enough, though most of the authors try to make the connection with their particular topic. This highlights the challenges of conducting research from a systems perspectives though I would argue that it is worth going this route rather than taking reductionist approaches to solving health problems, which can amount to 'looking for lost keys only where the light shines'.

The chapter on revenue collection and pooling arrangements in financing builds on the implicit assumption that government is effective, hence neglecting to address the possibility of government failure, to which low- and middle-income countries are of course not immune. Government inefficiency could hamper any arrangements for restructuring health financing. The authors talk about a redirected flow of funds from private to public (p. 88), but the assumption is that these funds will be used efficiently and equitably by government. They refer to the high cost of having many small schemes (p. 95), but do not look at the converse: the potentially high costs of having a large NHI, with the inevitable level of inefficiency and high administration costs associated with a public entity. But these are complex issues and those interested in this specific topic are encouraged to consult more specialised literature which expands on the intricacies involved.

I would recommend this book as a good starting point for anyone interested in learning more about health issues and health systems, particularly in developing countries. It is easy to read and thus well suited to a broad audience. It provides a basic working knowledge of the field, and offers comprehensive reference lists for the reader who wants to explore further. For me the particular value of the book is the way it clarifies theoretical issues to do with health systems, and I appreciated the authors' enthusiasm in recommending that all health-related research and policies adopt a systems-thinking approach to coping with health care problems.

Carmen Sue Christian Lecturer, Economics Department Economics and Management Science Faculty University of the Western Cape, Bellville, South Africa Correspondence: carmensue@live.com http://dx.doi.org/10.1080/0376835X.2012.730975 